\title{
Adaptive filter solution for processing lidar returns: optical parameter estimation
}

\author{
Francesc Rocadenbosch, Gregori Vázquez, and Adolfo Comerón
}

\begin{abstract}
Joint estimation of extinction and backscatter simulated profiles from elastic-backscatter lidar return signals is tackled by means of an extended Kalman filter (EKF). First, we introduced the issue from a theoretical point of view by using both an EKF formulation and an appropriate atmospheric stochastic model; second, it is tested through extensive simulation and under simplified conditions; and, finally, a first real application is discussed. An atmospheric model including both temporal and spatial correlation features is introduced to describe approximate fluctuation statistics in the sought-after atmospheric optical parameters and hence to include a priori information in the algorithm. Provided that reasonable models are given for the filter, inversion errors are shown to depend strongly on the atmospheric condition (i.e., the visibility) and the signal-to-noise ratio along the exploration path in spite of modeling errors in the assumed statistical properties of the atmospheric optical parameters. This is of advantage in the performance of the Kalman filter because they are often the point of most concern in identification problems. In light of the adaptive behavior of the filter and the inversion results, the EKF approach promises a successful alternative to present-day nonmemory algorithms based on exponential-curve fitting or differential equation formulations such as Klett's method. (C) 1998 Optical Society of America

OCIS codes: $010.0010,010.1290,010.3640$.
\end{abstract}

\section{Introduction}

The superior qualities of laser radars, or lidars, with regard to collimation, spatial resolution, and interaction capability with atmospheric species, when compared with those of conventional microwave radar systems or passive visible instrumentation, hold promise that lidars will be long-lasting alternative observation systems. In an atmospheric lidar, the emission of a short laser pulse is followed by the reception of some radiation scattered from atmospheric constituents such as molecules, aerosols, and clouds. The interaction of the incident radiation with these constituents changes the intensity and/or the wavelength, depending on the strength of this optical interaction and the concentration of the interacting species. Consequently, it is possible to retrieve information about the physical state of the atmosphere along the exploration beam path. ${ }^{1-3}$

The authors are with the Antennas, Microwaves, Radar and Optics Group, Department of Signal Theory and Communications, Universitat Politècnica de Catalunya, C/Sor Eulalia de Anzizu s/n., 08034 Barcelona, Spain.

Received 29 September 1997; revised manuscript received 8 April 1998.

0003-6935/98/307019-16\$15.00/0

(C) 1998 Optical Society of America
In particular, estimation of the atmospheric optical parameters, namely, extinction and backscatter, based on pulsed elastic-backscatter lidars (i.e., with no wavelength shift in reception) has been investigated in the literature. ${ }^{4-6}$ The single-scattering range-return power for an elastic-backscatter lidar system can be expressed $a^{2}$

$$
P(R)=\frac{A}{R^{2}} \beta(R) \exp \left[-2 \int_{0}^{R} \alpha(r) \mathrm{d} r\right],
$$

where $P(R)$ is the range-received power (W), $\beta(R)$ is the range-dependent volume backscatter coefficient of the atmosphere $\left(\mathrm{m}^{-1} \mathrm{sr}^{-1}\right), \alpha(R)$ is the rangedependent extinction coefficient $\left(\mathrm{m}^{-1}\right), R$ is the range (m), and $A$ is the system constant ( $\mathrm{W} \mathrm{m}^{3}$ ).

Until now, the inversion of lidar signals has been tackled mainly by using classic procedures such as the slope method, ${ }^{5}$ exponential-curve fitting, and Klett's method. ${ }^{6}$ Yet, all these methods assume simplifying and/or correlation hypotheses that limit the scope of the inversion results. These are discussed next.

In the case of the slope-method algorithm, ${ }^{5}$ the assumption of a homogeneous atmosphere is used to retrieve constant values $(\alpha, \beta)$ as estimates of the 
sought-after functions $\alpha(R)$ and $\beta(R)$. The key to the algorithm is a range-corrected function of the form

$$
S(R)=\ln \left[R^{2} P(R)\right],
$$

which enables us to find the extinction and backscatter coefficients from a linear regression of the form

$$
\min _{\alpha, \beta}\|S(R)-[\ln (A \beta)-2 \alpha R]\|^{2} .
$$

In the case of exponential-curve fitting, a similar approach is followed, but now the $R^{2}$-corrected function is defined without the logarithm as

$$
F(R)=R^{2} P(R),
$$

so that the norm minimization equivalent to expression (3) takes the form

$$
\min _{\alpha, \beta}\|F(R)-A \beta \exp (-2 \alpha R)\|^{2} .
$$

Historically, this kind of fitting was introduced later because it is nonlinear in $\alpha$ and must be solved by using numerical methods..$^{7,8}$

In a different category, the inversion of the rangedependent function $\alpha(R)$ is first solved by Klett's method. ${ }^{6}$ The method assumes a power-law correlation between the extinction and backscatter atmospheric profiles as follows:

$$
\beta(R)=B_{0}[\alpha(R)]^{\gamma},
$$

and it requires a guess of the correlation constant $\gamma$ $(0.67<\gamma<1)$ and a calibration at the far end of the inversion range interval in terms of $S_{m}=S\left(R_{\max }\right)$ and $\alpha_{m}=\alpha\left(R_{\text {max }}\right) \cdot 9,10$ Then the backward stable solution for $\alpha(R)$ becomes

$$
\alpha(R)=\frac{\exp \left[\left(S-S_{m}\right) / \gamma\right]}{\alpha_{m}{ }^{-1}+\frac{2}{\gamma} \int_{R}^{R_{m}} \exp \left[\left(S-S_{m}\right) / \gamma\right] \mathrm{d} r} .
$$

In spite of the fact that this algorithm is significantly superior to the slope method and the exponential-curve fitting (because the homogeneity approximation is not assumed), the accuracy of the inverted profile $\alpha(R)$ is limited by that of the calibration $\alpha_{m}$ and the correlation constant $\gamma$. For this reason the algorithm retrieves a representative of the family $\alpha\left(R, \gamma, \alpha_{m}\right)$ linked to the calibration pair $(\gamma$, $\alpha_{m}$ ), which is thought to be close to the true extinction profile $\alpha(R)$. In other words, the inversion of a range-dependent extinction profile from the return power is a many-to-one inversion problem (see Appendix B in Ref. 11), which can be solved only by adding appropriate $a$ priori information (e.g., calibrations along the observation path and physical constraints).

As we have seen, all these algorithms work with the present realization of the lidar return signal, so that correlation among past inverted returns remains unexplored. For example, in an elasticbackscatter pulsed lidar system, for each returnpower data stream received a new inversion, which is completely independent from those previously done, is performed.

One of the things that distinguishes the Kalman filter ${ }^{12-14}$ from nonmemory estimators such as those discussed above is the convenient way in which it accounts for any prior knowledge through a recursive process. As long as different power realizations are coming in, the filter updates itself, weighted by the imbalance between the a priori estimates of the optical parameters (i.e., past inversions) and the new ones. Thus the new estimation of the optical parameters, or the project-ahead step (a posteriori estimate), is improved based on a statistical minimum-variance criterion.

In recent and pioneering work, Rye and Hardesty ${ }^{15}$ and Lainiotis et al. ${ }^{16}$ have found applications of the Kalman filter to the estimation of the return power and the logarithm of power for incoherent backscatter lidar with multiplicative noise. ${ }^{17}$ Here we introduce an application of the filter to the solution of the inverse problem of joint estimation of the extinction and backscatter coefficients from the return power in an elastic-backscatter lidar.

This paper is structured as follows: In Section 2 the problem is formulated from a theoretical point of view in terms of a first adaptive filter based on an extended Kalman filter (EKF); Section 3 describes the underlying statistics of the atmospheric model assumed in terms of the state-noise covariance matrix of the EKF; Section 4 discusses two examples of joint inversion of extinction and backscatter profiles from elastic-backscatter simulated lidar return signals; and Section 5 reviews some of the results presented by tackling a first real application of the filter to the inversion of power returns from a biaxial elastic-backscatter 1-J Nd:YAG lidar system.

\section{Problem Formulation}

It is desirable now to study the feasibility of the derivation of the extinction and backscatter coefficients over the entire lidar inversion range. It is desired, then, to solve the functions $\alpha(R, t)$ and $\beta(R, t)$ that, under a minimum-mean-square-error criterion, best fit the observable power $P(R, t)$ at every time $t$. The term mean refers here to the ensemble average over time $t$. (Some revision of the EKF algorithm, along with the notation used below, is summarized in Appendix A.)

\section{A. State Vector}

Given the acquisition sampling rate of the system, $f_{s}$, and considering the two-way path of the lidar signal, the power time samples $P_{i}$ correspond to a spatial sampling period

$$
\Delta R=\frac{c}{2 f_{s}}
$$

Hence the spatial sampling points become

$$
R_{i}=R_{\text {min }}+(i-1) \Delta R, \quad i=1, \ldots, N,
$$


where $R_{\min }$ is some predetermined minimum range of the system (which is due to, for example, the minimum range of full overlap between the laser and the field of view of the receiving optics or some predefined minimum inversion range of interest).

The state vector to be estimated, $x_{k}$, is a decimated version of the extinction and backscatter functions $\alpha(R)$ and $\beta(R)$ over the whole lidar range. This is done in order to have more observables (the power samples from each observation cell) than variables to estimate (extinction and backscatter samples in the estimation cells), and, as a result, it yields an overdetermined system with enhanced observability. ${ }^{14,18}$ Furthermore, it can be shown ${ }^{14}$ that if (1) the system state vector is assumed to be a random constant, (2) the measurement sequence $z_{k}$ yields an overdetermined set of linear equations, and (3) the observation noise becomes negligible [i.e., the measurement noise covariance matrix of Eq. (A4), $\left.R_{k} \approx 0\right]$, then the filter's estimate depends more and more on current data (which are rich in new information) and less and less on past inversions. Under these circumstances the filter behaves like a deterministic least-squares estimator (which parallels the nonmemory approach), and its estimate becomes ${ }^{13}$

$$
\hat{x}_{k}=H_{k}^{-1} z_{k}
$$

where $H_{k}{ }^{-1}$ is the pseudoinverse matrix of $H_{k}$ [see relation (A6)]. Historically, this bridges the gulf with past formulations of the problem in the form of expression (5).

The model considers $N / M$ observation cells, where $M$ is the decimation ratio, so that the filter estimates $N / M$ extinction samples and $N / M$ backscatter samples. Then the effective sampling period becomes $M \Delta R$, which is $M$ times that of the return power. (For simplicity, assume that $N$ is a multiple of $M$.) Mathematically, this can be expressed as

$$
\begin{array}{ll}
\alpha_{i}=\alpha\left(R_{\text {min }}+(i-1) M \Delta R\right), & i=1, \ldots, \frac{N}{M}, \\
\beta_{i}=\beta\left(R_{\text {min }}+(i-1) M \Delta R\right), & i=1, \ldots, \frac{N}{M} .
\end{array}
$$

From these two halves of $N / M$ elements, we form the state vector to be estimated:

$$
x_{k} \equiv\left(\begin{array}{llllllll}
\alpha_{1} & \alpha_{2} & \cdots & \alpha_{N / M} & \beta_{1} & \beta_{2} & \cdots & \beta_{N / M}
\end{array}\right)^{\mathrm{T}},
$$

where the subscript $k$ is a reminder of the discrete time $t_{k}$.

The nonstationarity or the dynamics of the state vector is described by the transition matrix $\Phi_{k}$ [see Eqs. (A1) and (A19)] and the state-noise covariance matrix $Q_{k}$ [Eq. (A2)]. The former represents how the state vector projects ahead from time $t_{k}$ to time $t_{k+1}$, and the latter gives the filter key information about the underlying statistics of each component of the state vector (the optical parameters under study). Formulation of the system equations in terms of the transition matrix $\Phi_{k}$ and the state-noise covariance matrix $Q_{k}$ gathers all the information the filter knows about the atmospheric model. This is tackled in Section 3.

\section{B. Measurement Equation}

If, according to Eq. (9), each power sample corresponds to a spatial increment $\Delta R$, so that $P_{i}=P\left(R_{i}\right)$, and a rectangle approximation is used to compute the transmittance term of Eq. (1), the observable power samples become

$$
\begin{gathered}
P_{1}=\frac{A}{R_{1}{ }^{2}} \beta_{1} \exp \left(-2 \alpha_{1} R_{\min }\right), \\
\vdots \\
P_{M}=\frac{A}{R_{M}{ }^{2}} \beta_{1} \exp \left\{-2 \alpha_{1}\left[R_{\min }+(M-1) \Delta R\right]\right\}, \\
P_{M+1}=\frac{A}{R_{M+1}{ }^{2}} \beta_{2} \exp \left\{-2 \alpha_{1}\left[R_{\min }+(M-1) \Delta R\right]\right. \\
\left.-2 \alpha_{2} \Delta R\right\}, \quad \vdots \\
P_{N}=\frac{A}{R_{N}{ }^{2}} \beta_{N / M} \exp \left\{-2 \alpha_{1}\left[R_{\min }+(M-1) \Delta R\right]\right. \\
\left.\quad-2 \sum_{i=2}^{N / M} \alpha_{i} M \Delta R\right\} .
\end{gathered}
$$

The $R^{2}$-corrected version [Eq. (4)] of this set of $N$ equations builds the measurement vector $z_{k}$ :

$$
z_{k} \equiv\left[\begin{array}{llll}
F_{1}\left(x_{k}\right) & F_{2}\left(x_{k}\right) & \cdots & F_{N}\left(x_{k}\right)
\end{array}\right]^{\mathrm{T}} .
$$

By using $F(R)$ rather than $P(R)$, we reduce the dynamic margin of $z_{k}$ and, consequently, numerical errors are reduced as we cycle through the Kalman loop [Eqs. (A13)-(A17)]. At the far ranges, however, amplification of quantization noise generated during the analog-to-digital conversion might become significant and must be accounted for in a description of the statistics of the observation noise.

Equations (13)-(16) define the overdetermined set of equations discussed in Subsection 2.A that relates the measurement vector $z_{k}$ to the $a$ priori estimate of the state vector, $\hat{x}_{k}^{-}$.

From Eqs. (12) and (17), the $N \times(2 N / M)$ observation matrix $H_{k}$ can be computed by splitting it in two $N \times(N / M)$ submatrices of the form $H=\left(H_{1} H_{2}\right)$, where

$$
H_{i j}^{(1)}=\left.\frac{\partial F_{i}}{\partial \alpha_{j}}\right|_{x=\hat{x}_{k}^{-}}, \quad H_{i j}^{(2)}=\left.\frac{\partial F_{i}}{\partial \beta_{j}}\right|_{x=\hat{x}_{k}^{-}} .
$$


This yields

$$
\begin{aligned}
& H_{1}=\left[\begin{array}{ccccc}
-2 R_{\min } F_{1} & 0 & 0 & \cdots & 0 \\
-2\left(R_{\min }+\Delta R\right) F_{2} & 0 & 0 & \cdots & 0 \\
\vdots & \vdots & \vdots & \cdots & \vdots \\
-2\left[R_{\min }+(M-1) \Delta R\right] F_{M} & 0 & 0 & \cdots & 0 \\
-2\left[R_{\min }+(M-1) \Delta R\right] F_{M+1} & -2 \Delta R F_{M+1} & 0 & \cdots & 0 \\
\vdots & \vdots & \vdots & \cdots & \vdots \\
-2\left[R_{\min }+(M-1) \Delta R\right] F_{N} & -2 M \Delta R F_{N} & -2 M \Delta R F_{N} & \cdots & -2 M \Delta R F_{N}
\end{array}\right]_{N \times(N / M)} \\
& H_{2}=\left(\begin{array}{ccccc}
\frac{F_{1}}{x_{N / M+1}} & 0 & 0 & \cdots & 0 \\
\frac{F_{2}}{x_{N / M+1}} & 0 & 0 & \cdots & 0 \\
\vdots & \vdots & \vdots & \cdots & \vdots \\
\frac{F_{M}}{x_{N / M+1}} & 0 & 0 & \cdots & 0 \\
0 & \frac{F_{M+1}}{x_{N / M+2}} & 0 & \cdots & 0 \\
\vdots & \vdots & \vdots & \cdots & \vdots \\
0 & 0 & 0 & \cdots & \frac{F_{N}}{x_{2 N / M}}
\end{array}\right)
\end{aligned}
$$

where $H_{1}$ and $H_{2}$ are evaluated at the $a$ priori estimate $\hat{x}_{k}^{-}$.

From the structure of $H_{1}$ and $H_{2}$, it emerges that the formulation of the inversion problem involves, however, a trade-off between larger decimation ratios $(M)$ and model accuracy. From the point of view of $M$, if one compares the EKF formulation with the classical exponential-curve-fitting counterpart of expression (5), the exponential fitting algorithm works with a very large value of $M$, equal to the length of the inversion interval, which is, in turn, formed by a single inversion cell. Hence the larger the $M$, the more robust the system of Eq. (10) against observation noise and the better the regression results [this is best seen by the products $M \Delta R F_{i}$ in Eq. (19), which become larger for larger $M]$. Another advantage of increasing $M$ is the enhancement of the filter's sensitivity to low return powers and large values of $R_{\text {min }}$. Usually, large differences between the weight factors $-2\left[R_{\min }+(M-\right.$
1) $\Delta R]$ and $-2 M \Delta R$ are not desirable, since then thetrajectory of the filter would be dominated by the estimation of the first cell. Yet, the most risky drawback for large $M$ arises from the deterioration of the filter's model [Eqs. (13)-(16)]: Although the equivalent sampling period of the optical parameters in the filter's model is $M \Delta R$, the true atmospheric spacing $\Delta R^{\prime}$ is differential in nature. For the time being, we assume the simplification $\Delta R^{\prime}=$ $\Delta R$ in the atmospheric optical profile, so that such modeling errors are neglected. In other words, the atmosphere is assumed homogeneous inside any observation cell. Although much research is being done in this field, which is far from the scope of this study, a sensible solution might be achieved by combining the results of an array of $M$ cooperative filters or, perhaps, by using nonuniform spacing in the formulation of the problem, depending on the atmospheric situation at hand. 
Lidar measurements are corrupted mainly by Gaussian additive observation noise $v_{k}$. Assuming that $\Delta R^{\prime}=\Delta R$ and that the observation noise along the inversion range can be approximated by rangedependent stationary electronic thermal noise (Gaussian additive noise with variance $\sigma_{r}^{2}$ ), the observation noise covariance matrix is computed as

$R_{k}=E\left(v_{k} v_{k}{ }^{\mathrm{T}}\right)=\left[\begin{array}{ccc}\sigma_{r}{ }^{2}\left(R_{1}\right) R_{1}{ }^{4} & \cdots & 0 \\ \vdots & \ddots & \vdots \\ 0 & \cdots & \sigma_{r}{ }^{2}\left(R_{N}\right) R_{N}{ }^{4}\end{array}\right]$.

This assumes that electronic noise dominates observation noise. Following Refs. 17 and 19, the rangedependent noise variance can be written as

$$
\sigma_{r}^{2}(R)=a\left[P(R)+P_{\text {back }}\right]+b,
$$

where $P(R)$ is the range return power defined in Eq. (1), $P_{\text {back }}$ is the background power from any other interfering source (for example, the Sun), and $a$ and $b$ are constants that depend only on specific parameters of the receiving system. The first term accounts for the contributions of the signal-induced shot noise to the total noise, and the second one merges into the variable $b$ the contributions of both dark-current shot noise and thermal noise. [Note that $\sigma_{r}^{2}(R)$ has units of square volts or square watts, depending on whether equivalent noise is computed at the receiver's output or input.

In instances where other sources of measurement noise are present (e.g., $R^{2}$-amplified quantization noise), one can increase pertinent terms along the main diagonal of $R_{k}$ to accommodate such an extra variance. Nonstationary noise can be tackled by recomputing $R_{k}$ at each succeeding step of the filter, and colored noise (such as synchronized flash-lamp interferences) can be modeled by also using elements off the main diagonal of $R_{k}$ (see also Ref. 14 for further insight).

\section{Atmospheric Model for the Extended Kalman Filter}

In their most general form, extinction and backscatter optical parameters are nonlinearly related. Unless microscale analysis is considered and plenty of boundary calibrations from balloon-borne instrumentation or other cooperative systems are given, the struggle to model physically the temporal and spatial evolution of the optical parameters leads to awkward and cumbersome results. A more convenient alternative is to try to model the macroscopic effects on them, rather than the underlying microphysical parameters. This is done by using the time-space stochastic correlation model sketched in Fig. 1. Each optical component (extinction and backscatter) of an inversion cell is modeled as a stochastic process having both temporal and spatial correlation. Each output branch represents one component of the state vector $x_{k}$, and the vector noise process $w_{k}$ is formed by spatially correlated components at the output of the

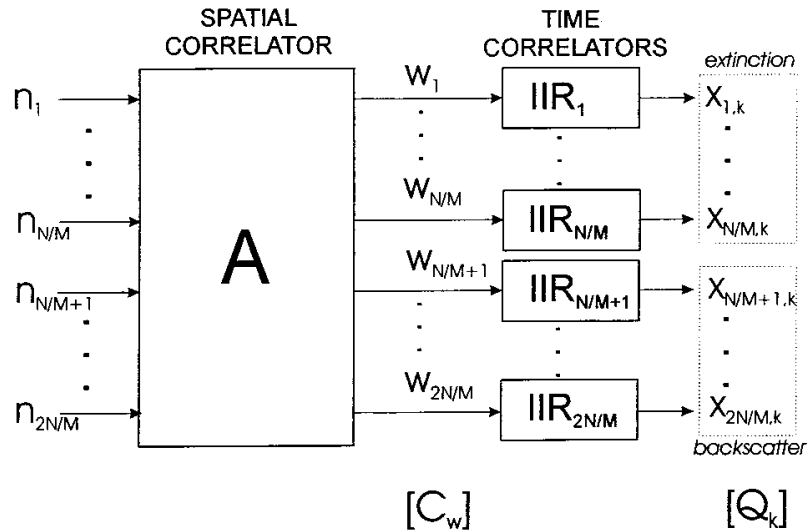

Fig. 1. Time-space EKF correlation model.

linear system A. The atmospheric model is driven by an array of white-noise uncorrelated processes.

\section{A. Temporal Correlation}

Temporal correlation is perhaps the most attractive advantage of the EKF lidar inversion approach over the nonmemory solutions of expressions (3) and (5) and Eq. (7). This advantage comes from telling the filter that it should improve its projection steps based on the fact that the atmosphere usually has a long correlation time and that, consequently, swift changes in any optical parameter are not possible. Temporal correlation is achieved by modeling each component of the state vector $x_{k}$ (with $k$ the discrete time) as a Gauss-Markov process. (To simplify the notation, we define the Markovian process $y_{k}$ as the $i$ th component of vector $x_{k}$, so that $y_{k}=x_{i, k}$.)

The Gauss-Markov process, ${ }^{14}$ which is often called Markovian noise, is zero-mean low-pass filtered Gaussian noise, whose autocorrelation function is given by

$$
R_{y}(\tau)={\sigma_{m}}^{2} \exp (-\delta|\tau|),
$$

where $\sigma_{m}^{2}$ is the power of the process $y_{k}$ and $\delta$ is the 3 -dB cutoff frequency of the low-pass coloring filter (IIR boxes in Fig. 1, where IIR stands for infinite impulse response).

The discrete-time equation of the process can be written in the form of an autoregressive movingaverage scalar process ${ }^{20}$ as

$$
y_{k+1}=\exp \left(-1 / L_{c}\right) y_{k}+w_{k},
$$

where $y_{k}$ and $w_{k}$ are the Markovian and white sequences, respectively, and $L_{c}$ is the temporal correlation length, defined as

$$
L_{c}=1 / \delta,
$$

where $L_{c}$ has units of samples [the spatial period has already been defined in Eq. (8)].

Finally, Eqs. (24) and (25) enable us to express the 
STATE VECTOR $x_{k}$

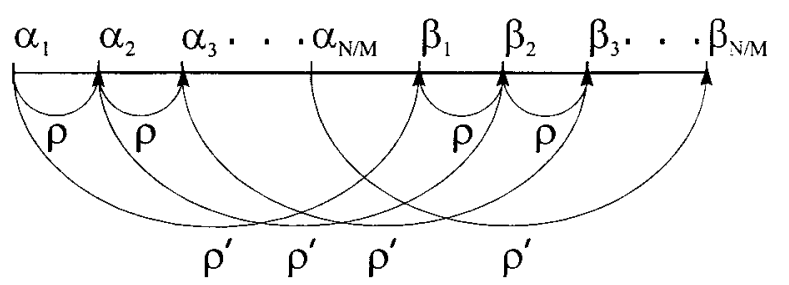

Fig. 2. Spatial correlation graph of the state-vector components.

state-vector transition matrix associated with Eq. (A19) as

$$
\Phi_{k}=\exp \left(-1 / L_{c}\right) \mathbf{I}
$$

where $\mathbf{I}$ is a $(2 N / M) \times(2 N / M)$ identity matrix and we have used the simplifications that $\Phi_{k}$ is constant over time $t_{k}$ and that $L_{c}$ is the same for all the cells along the lidar exploration path. With $\Phi_{k}$ a matrix, $w_{k}$ also becomes a vector, whose $2 N / M$ components represent white sequences at each time $t=t_{k}$.

In practice, Markovian noise is responsible for the time drift of the actual value of the optical parameters being estimated by the filter at each projection step. For this reason it is useful to define an intensity parameter $p$ that enables us to adjust the power of the Markovian process or, equivalently, to link the driving Gaussian noise power to the amplitude change caused in an optical parameter. From Eqs. (23) and (25) and Ref. 14, the Gaussian noise standard deviation $\sigma_{w}$ and the Markovian one, $\sigma_{m}$, can be related as

$$
\sigma_{w}=\sigma_{m} \sqrt{1-\exp \left(-2 / L_{c}\right)}
$$

A comprehensive collection of histograms for a given $\sigma_{m}$ have shown that over $95 \%$ of the Markovian amplitudes distribute between $\pm 2.5 \sigma_{m}$. With that in mind, the white-noise strength $\sigma_{w}=\sigma_{\alpha_{i}}$ that is needed to cause a $p$-per-one change in the amplitude of a vector component of $x_{k}$ (let it be $\alpha_{i}$ ) over a correlation length $L_{c}$ becomes

$$
\sigma_{\alpha_{i}}=\frac{p}{2.5} \alpha_{i} \sqrt{1-\exp \left(-\frac{2}{L_{c}}\right)}, \quad i=1, \ldots, \frac{N}{M} \text {. }
$$

\section{B. Spatial Correlation}

Contrary to what happened with nonmemory algorithms, where analytical correlation relations were assumed [for example, homogeneity for the slope and exponential-curve-fitting algorithms of expressions (3) and (5), respectively, or the power-law correlation of Eq. (6) for Klett's method], the approach presented here is based on the correlation graph of Fig. 2. It paves the way for the introduction of loose stochastic relations among the sought-after optical parameters instead of tight analytical ones. It seems sensible to guess that, for example, any extinction change in a particular cell will, in turn, influence variations not only in the in-cell backscatter component but also in the extinction and backscatter components of its neighboring cells. The underlying physical phenomenon being the cause, the changes may well extend over several cells. From the correlation graph of Fig. 2, one can build the white-noise state-vector covariance matrix as follows:

$$
C_{w}=\left[\begin{array}{ll}
C_{\alpha \alpha} & C_{\alpha \beta} \\
C_{\beta \alpha} & C_{\beta \beta}
\end{array}\right]
$$

These block matrices can be developed as

$$
\begin{gathered}
C_{\alpha \alpha}=\left[\begin{array}{cccc}
\sigma_{\alpha_{1}}{ }^{2} & \rho \sigma_{\alpha_{1}} \sigma_{\alpha_{2}} & \cdots & \rho^{n-1} \sigma_{\alpha_{1}} \sigma_{\alpha_{n}} \\
\cdots & \sigma_{\alpha_{2}}{ }^{2} & \cdots & \rho^{n-2} \sigma_{\alpha_{2}} \sigma_{\alpha_{n}} \\
\cdots & \cdots & \cdots & \cdots \\
\cdots & \cdots & \cdots & \sigma_{\alpha_{n}}^{2}
\end{array}\right], \\
C_{\alpha \beta}=\left[\begin{array}{cccc}
\rho^{\prime} \sigma_{\alpha_{1}} \sigma_{\beta_{1}} & \rho^{\prime} \rho \sigma_{\alpha_{1}} \sigma_{\beta_{2}} & \cdots & \rho^{\prime} \rho^{n-1} \sigma_{\alpha_{1}} \sigma_{\beta_{n}} \\
\cdots & \rho^{\prime} \sigma_{\alpha_{2}} \sigma_{\beta_{2}} & \cdots & \rho^{\prime} \rho^{n-2} \sigma_{\alpha_{2}} \sigma_{\beta_{n}} \\
\cdots & \cdots & \cdots & \cdots \\
\cdots & \cdots & \cdots & \rho^{\prime} \sigma_{\alpha_{n}} \sigma_{\beta_{n}}
\end{array}\right],
\end{gathered}
$$

where $C_{\beta \alpha}=C_{\alpha \beta}, C_{\beta \beta}$ is the same as $C_{\alpha \alpha}$ but with $\beta$ and $\alpha$ permuted, $\rho$ is the correlation coefficient between one cell and the next one along the beam path (which is due to the physical continuity of the atmosphere), $\rho^{\prime}$ is the in-cell extinction-to-backscatter correlation coefficient, and $\sigma_{i}$ has been defined above in Eq. (28). On the condition that $|\rho|<1,\left|\rho^{\prime}\right|<1$, it can be proved that the graph of Fig. 2 does represent a covariance matrix.

Assuming that temporal and spatial correlation processes are independent, the Markovian noise state-vector covariance matrix (i.e., the sought-after covariance matrix for the atmospheric model given to the filter, $Q_{k}$ ) can be computed from Eqs. (27), (29), (30), and (31) as

$$
Q_{k}=\frac{C_{w}}{1-\exp \left(-2 / L_{c}\right)},
$$

where $Q_{k}$ and $C_{w}$ are basically the same except for a scaling factor, which could, in turn, be merged into an equivalent intensity parameter $p^{\prime}$.

Although the inversion of wind fields is far from the objective of this study, this independent hypothesis between temporal and spatial correlation is, however, doubtful in situations with a significant radial wind component (i.e., the wind component along the exploration path). In these instances radial wind strongly correlates both space and time fluctuations along the line of sight. Here one might consider only the spatial correlation of Fig. 1 (i.e., $Q_{k}=C_{w, k}$ ), but this time a variant one, since the observation cells along the path become progressively affected by different correlation links as time goes on. In addition, boosting elements off the main diagonal would tell the filter of a significant increase in the correlation among neighboring cells. In any case the possibility of modeling nonstationary statistics in $Q_{k}$ by resetting it at each succeeding step of the filter offers a wide span of attractive possibilities yet to be investigated. 


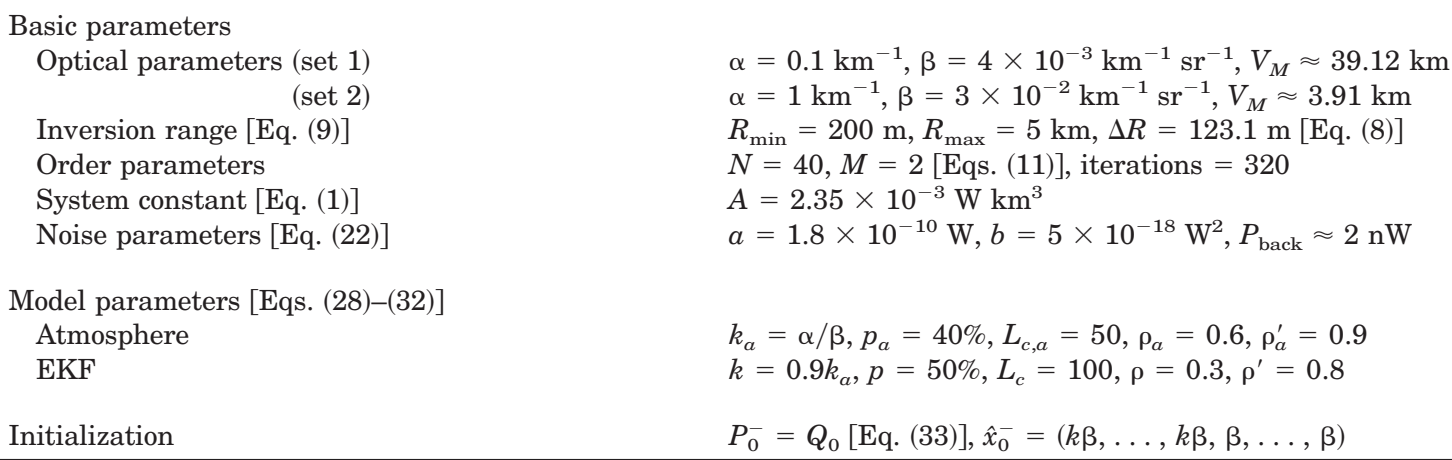

Usually, the state-noise covariance matrix of the EKF, $Q_{k}$, is the most difficult input to assess, since its atmospheric counterpart $Q_{k, a}$ (the subscript $a$ refers to atmospheric) is unknown. The problem of finding good models for $Q_{k}$ has sometimes been tackled by using a partitioned approach, ${ }^{16,21,22}$ where the unknowns are merged into a vector of random variables $\Theta=\left(\theta_{1} \cdots \theta_{P}\right)$ with known or assumed $a$ priori probability density functions (consider, for example, Ref. 16). This approach yields a bank of EKF's working in parallel, each matched to an appropriate value of $\theta_{i}$, so that the overall vector $\Theta$ spans the space of unknown parameters constrained by their related possible values. In theory, joint estimation of the extinction and backscatter parameters $\Theta$ would virtually apply to all the elements of $Q_{k, a}$ plus, possibly, the equivalent intensity parameter $p^{\prime}$. In practice, this would involve a large array of filters that would probably exceed the framework of intelligent and selforganizing systems, and hence it would certainly prevent a straightforward formulation of the study. For this reason, at this first stage, estimation with a single EKF is preferred in this experimental work, even though this is done at the expense of larger modeling errors and, hence, worse performance. These model uncertainties justify a formulation of the a priori error covariance matrix as

$$
P_{0}^{-}=\mu Q_{0}, \quad \mu \geq 1 .
$$

With regard to the atmospheric model, the simulations have used a set of parameters $Q_{k, a}, L_{c, a}$, and $p_{a}$ different from those given to the EKF model, $Q_{k}$, $L_{c}$, and $p$, to test the performance of the filter under modeling errors. Eigenvalue decomposition is used to compute the linear correlator (A in Fig. 1) and the power of the white-noise uncorrelated sequences $\left(n_{1} \cdots n_{N / M}\right)$, which are the driving inputs of the atmospheric simulator. ${ }^{14}$

\section{Simulation Results}

Through extensive simulation and simplified conditions, joint estimation of extinction and backscatter simulated profiles from elastic-backscatter lidar return signals have been inverted by using the formulation presented above. Next, two simulation sets are discussed; the first one (Figs. 3-7) corresponds to a good-visibility scene, and the second one (Figs. 8-10) corresponds to moderate-visibility conditions. Simulation parameters are summarized in Table 1. First, we give a brief outline of the choice of statistical parameters.

The choice of $\rho_{a}$ and $\rho_{\alpha}^{\prime}$ is based on cross-examined time-space plot sets of synthesized power return signals with nonwindy time-space real observations. Good agreement between typical real data sets and simulated ones has usually been achieved for large values of $\rho_{a}^{\prime}$ (typically between 0.8 and 0.9 ) and medium values of $\rho_{a}$ (typically between 0.3 and 0.7 ). The former result is also in accordance with Eq. (6), where $\gamma=1$ is equivalent to $\rho_{a}^{\prime} \rightarrow 1$. As for the latter, it has been found that $\rho_{a}$ values close to unity are not advisable because they yield stiff spatial profiles that are so correlated that it is difficult to accommodate even moderate heterogeneities along the lidar path. It has also been found that the intensity parameter $p_{a}$ is the most critical of all and that it must be adjusted to each particular scene. As a rule of thumb for low atmospheric extinctions, measurement of the fluctuations in the range-corrected power has yielded acceptable estimations of $p . \quad L_{c}$ is usually determined from rough visual estimation.

The first simulated set is related to a mean visibility of $V_{M}=39.12 \mathrm{~km}$. Such visibility conditions are typical of standard clear to exceptionally clear air. From Refs. 23 and 24 and under the approximation of a homogeneous atmosphere, the visibility parameter can roughly be linked to the atmospheric optical parameters $\alpha=0.1 \mathrm{~km}^{-1}$ and $\beta=4 \times 10^{-3} \mathrm{~km}^{1} \mathrm{sr}^{-1}$ or, equivalently, $k_{a}=25 \mathrm{sr}$ and $\beta=4 \times 10^{-3} \mathrm{~km}^{-1} \mathrm{sr}^{-1}$, where $k_{a}$ is the extinction-to-backscatter ratio indicated in Table 1 and the subscript $a$ refers to atmosphere. Hence one can without distinction talk about visibility or homogeneous atmospheric optical parameters $(\alpha, \beta)$.

To simulate an inhomogeneous profile approximately related to the visibility $V_{M}$, the simulator computes a range-dependent hump-shaped backscatter profile with mean $\beta$, such as the one shown in Fig. $3(\mathrm{a})$. For other visibility margins, the profile is scaled accordingly. In this way it is ensured that the synthesized profile is always approximately related to the average visibility desired. We computed the 

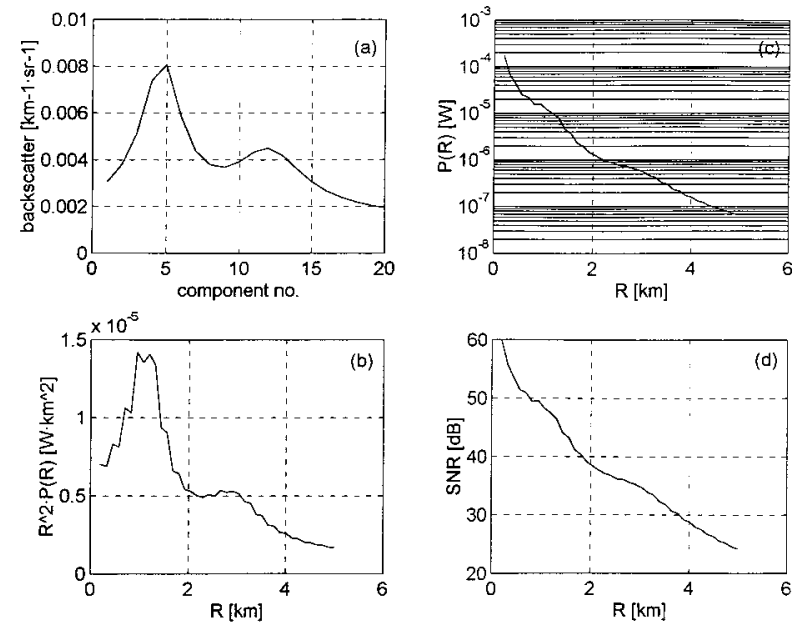

Fig. 3. (Set 1) initial state of the simulation: (a) synthesized backscatter profile, (b) range-corrected return power, (c) return power as received by the lidar, (d) associated SNR.

range-dependent extinction profile after the backscatter by reusing the extinction-to-backscatter ratio $k_{a}$. From the extinction and backscatter initial profiles just computed, the lidar range-return power of Eq. (1) follows as shown in Figs. 3(b) and 3(c). To reduce the order of the filter, the physical problem has been discretized by using $N=40$ and $M=2$, which means $N=40$ power samples and $N / M=20$ observation cells. Since each cell is defined by both its extinction and backscatter parameters, there are 40 state-vector components, 20 for each optical parameter.

To compute the observation noise, the simulator uses electrical and optical parameters from an elastic-backscatter lidar of the Polytechnic University of Catalonia in Barcelona, Spain (system specifications are given in Section 5) to assess realistic noise parameters in Eq. (22). They are representative of a typical tropospheric lidar system (see Table 1) and yield the range-dependent signal-to-noise ratio (SNR) of Fig. 3(d).

The atmospheric behavior was simulated by using the simplified model described in Section 3 and the model parameters of Table 1 . As for the EKF, a slightly mismatched model is input, so that, for example, the extinction-to-backscatter ratio is underestimated by $10 \%$, the temporal correlation length is doubled, and the spatial correlation coefficients are changed as indicated in Table 1 . These modeling errors translate into $Q_{k, a} \neq Q_{k}$, as suggested in Subsection 3.B. Since we are particularly concerned about the performance of the filter under different visibility conditions and atmospheric modeling errors, the simplification in which there are no mismatches in the model of $R_{k}$, so that both the observables and the filter share the same covariance matrix, has been assumed. This can be justified because $Q_{k, a}$ is always the hidden parameter of the atmosphere, whereas $R_{k}$ can ultimately be measured from the lidar system.

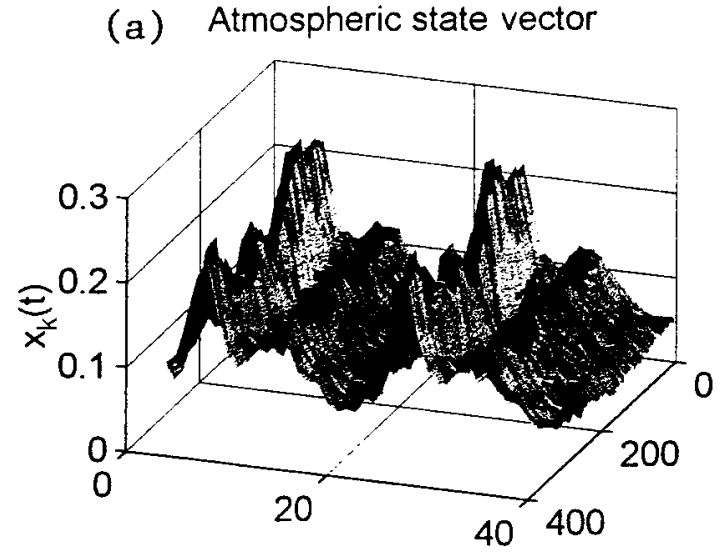

(b) Estimated state vector

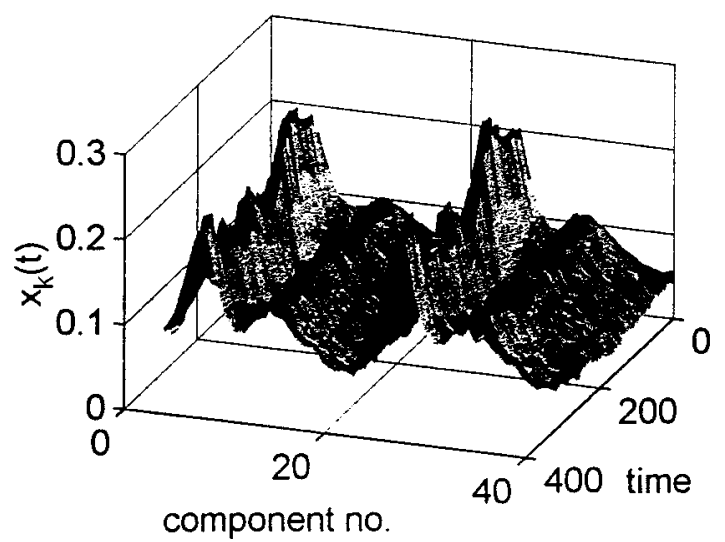

Fig. 4. (Set 1) time-space evolution of the extinction and backscatter profiles: (a) synthesized atmospheric optical parameters (extinction and backscatter), (b) EKF inverted optical parameters.

The initialization of the filter, $\hat{x}_{0}^{-}$, may come from any of the methods discussed in Section 1; in particular, Eq. (7) would yield the best approximation. Yet, to test the performance of the filter, it has been initialized in the simplest possible way by using a constant homogeneous profile for the extinction and backscatter components of the state vector, as indicated in Table 1. Since each simulation run takes 320 iterations, the filter depends more and more on the measurements and less and less on the initial state.

As time goes on, the actual measurement data (observables) received for any particular sample run change according to the atmospheric state model given by $\Phi_{k}$ and $Q_{k, a}$, so that slowly varying changes in both the extinction and backscatter profiles are accommodated. The filter keeps track of the timevarying nature of the observables from the beginning. Figures 4 and 5 illustrate the time evolution of the atmospheric model along with the EKF state-vector components. Recall that components 1-20 represent the extinction coefficient and components 21-40 represent the backscatter coefficient along with the observation cells, so that if one reads by cells, the first one comprises components 1 and 21 , the second one 
(a) Atmospheric state vector

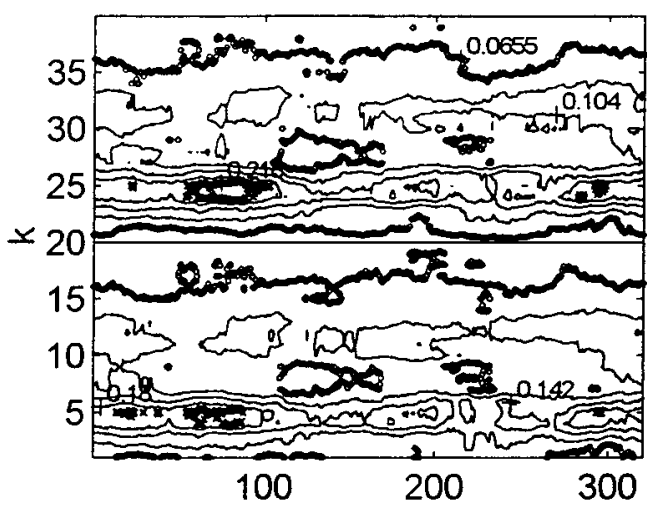

(b) Estimated state vector

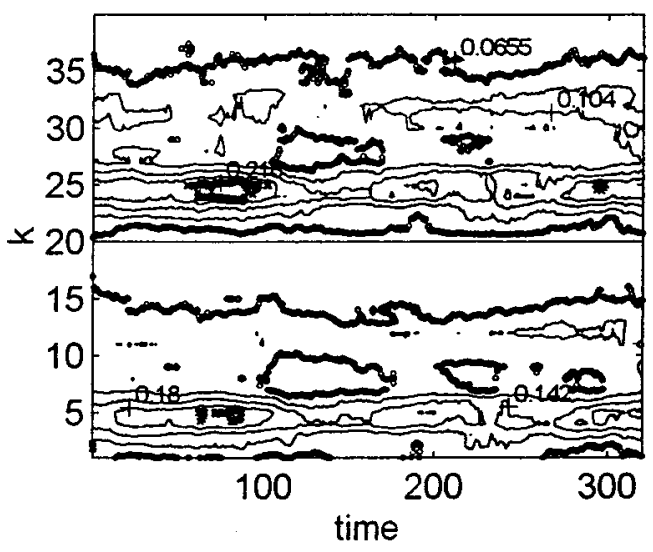

Fig. 5. (Set 1) contour plots of Fig. 4 showing very good correlation between the time-space evolution of the atmospheric optical parameters and the inverted ones: (a) synthesized atmospheric optical parameters, (b) EKF inverted optical parameters.

comprises components 2 and 22 , and so on. The temporal evolution of the mountains in Fig. 4 is caused by the Markovian noise. Spatially, with $\rho_{a}^{\prime}=$ 0.9 the in-cell extinction-to-backscatter correlation is so high that the two halves of each plot look virtually alike (note that for illustrative purposes the backscatter half has been rescaled by $k_{a}$ ).

Convergence of the EKF from the homogeneous initial profile to something close to a real profile can easily be tracked by monitoring the trace of the error covariance matrix $P_{k}$ [see Eq. (A18) in Appendix A and Section 5 for further insight]. Since $P_{k}$ informs the filter about the expected error that it is committing at each time $t_{k}$, a good convergence criterion is whether $P_{k}$ has reached a constant value. In the plots presented, as is always the case, the shape of the estimated profiles is retrieved fast, but their magnitudes (especially the extinction one) take some more time to settle. In the simulations the trace of $P_{k}$ settles by iteration 150 , although after iteration 50 most details from the true atmospheric profile at short ranges are recovered quite well.

Figure 5 is a contour plot of Fig. 4 representing isoextinction and isobackscatter curves (scaled by $k_{a}$
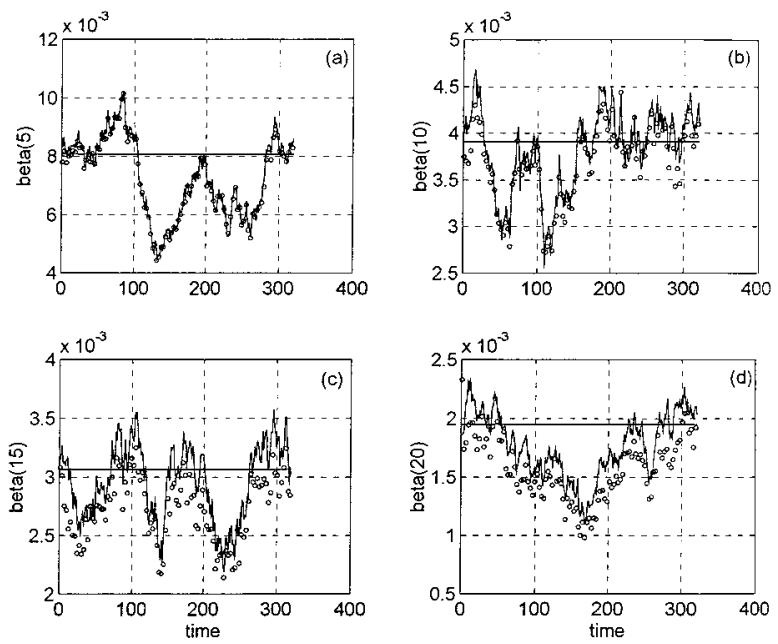

Fig. 6. (Set 1) temporal evolution of the backscatter profiles in four representative observation cells along the lidar beam path: (horizontal lines) starting backscatter values for the atmospheric simulator, (solid curves) atmospheric backscatter evolution, (circles) EKF estimates.

for illustrative purposes) along time for both the atmospheric and the estimated state vector. Both contours look virtually alike after the 50th iteration except for some slight deterioration in the far-range extinction components of the atmosphere (components 10-20), where the EKF performs more poorly. This, however, can easily be justified by the progressive reduction in the SNR of Fig. 3(d) for increasing ranges.

From the point of view of the time-space correlation models introduced in Subsections 3.A and 3.B, Fig. 6 compares the time evolution of the EKF estimates in four observation cells successively farther along the lidar exploration range (cells 5, 10, 15, and 20 located at $1307.7,2538.5,3769.2$, and $5000 \mathrm{~m}$, respectively) with their true atmospheric counterparts. The atmospheric backscatter evolution is denoted by solid curves, and the filter's estimates are given by small circles. The filter follows the random drift of each cell fairly well in all the cases, but whereas Figs. 6(a) and 6(b) show the best-fitted cells, Figs. 6(c) and 6(d) show some slight underestimation of the atmospheric backscatter. Horizontal solid lines indicate the initial backscatter value in each cell before the atmospheric simulator starts. These values correspond to the 5 th, 10th, 15th, and 20th components of Fig. 3(a). As expected from the temporal correlation model formulation of Subsection 3.A, Markovian noise translates into a slow temporal drift of the backscatter figure. For example, the atmospheric temporal correlation length $\left(L_{c, a}=50 \mathrm{sam}\right.$ ples) is best seen in Figs. 6(a) and 6(c) (solid curves). Thus, in Fig. 6(c), increasing and decreasing slopes last for approximately 50 samples on average, and the same happens in Fig. 6(a) except that now the plot includes some kind of horizontal interval. From the point of view of the spatial correlation, one has to compare all the plots. Since $\rho_{a}=0.6$ and each plot 

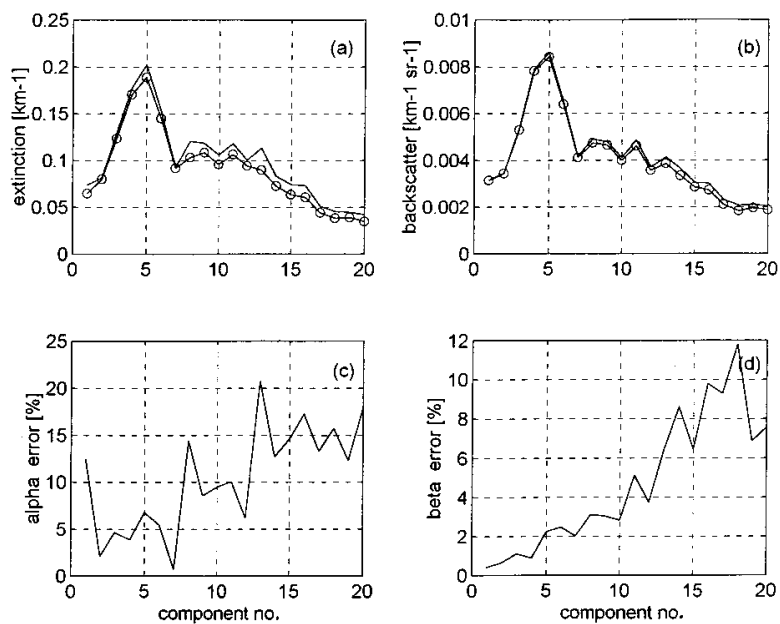

Fig. 7. (Set 1) Inversion results after 320 iterations: (a) final atmospheric extinction (solid curve), final EKF extinction estimates (circles); (b) final atmospheric backscatter (solid curve), final EKF backscatter estimates (circles); (c) extinction relative inversion error; (d) backscatter relative inversion error.

represents the behavior of a cell that is five cells apart from the others, $\rho_{a}{ }^{5} \approx 0.08$ means poor correlation among them.

Finally, Fig. 7 compares the final state of the atmospheric extinction and backscatter components with the EKF estimates and their relative inversion errors, with successful results. As expected, inversion errors increase in the far range (components 10-20), since these observation cells undergo the worst SNR's. Everything considered, inversion errors seldom exceed $20 \%$ and $10 \%$ for the extinction and backscatter components, respectively, for SNR's of approximately $25 \mathrm{~dB}$ at $5 \mathrm{~km}$. Therefore, as far as noise is concerned, a SNR at the receiver output (i.e., as a ratio of voltages) above $25 \mathrm{~dB}$ at $5 \mathrm{~km}$ seems enough to ensure good performance of the filter in the example.

The second simulation set is ruled by a mean visibility $V_{M}=3.91 \mathrm{~km}$, which is typical of a turbid atmosphere or moderate haze. From Refs. 23 and 24 , this visibility can roughly be linked to $\alpha=1 \mathrm{~km}^{-1}$ and $\beta=3 \times 10^{-2} \mathrm{~km}^{-1} \mathrm{sr}^{-1}$ or, equivalently $k_{a}=33$ sr and $\beta=3 \times 10^{-2} \mathrm{~km}^{-1} \mathrm{sr}^{-1}$. All the other basic model parameters were set as indicated in Table 1. Simulation results are reproduced in Figs. 8-10.

The synthesis of the atmospheric optical parameters from the visibility margin $V_{M}$ follows the procedure discussed above, so that the shape of the synthesized backscatter profile is exactly the same as that in Fig. 3(a), but it is conveniently scaled to the new value of visibility. Compared with Fig. 3(d), the SNR plot would decrease in a linear fashion (in the $\log$ grid) from $67.5 \mathrm{~dB}$ at $R_{\min }=200 \mathrm{~m}$ down to -31.0 $\mathrm{dB}$ at $R_{\max }=5 \mathrm{~km}$, where $\mathrm{SNR}$ is defined as a ratio of voltages at the received output. If one follows a closeness criterion between both the true and estimated profiles, SNR $>25 \mathrm{~dB}$ yields extinction and backscatter relative errors below $20 \%$ and the useful (a) Atmospheric state vector

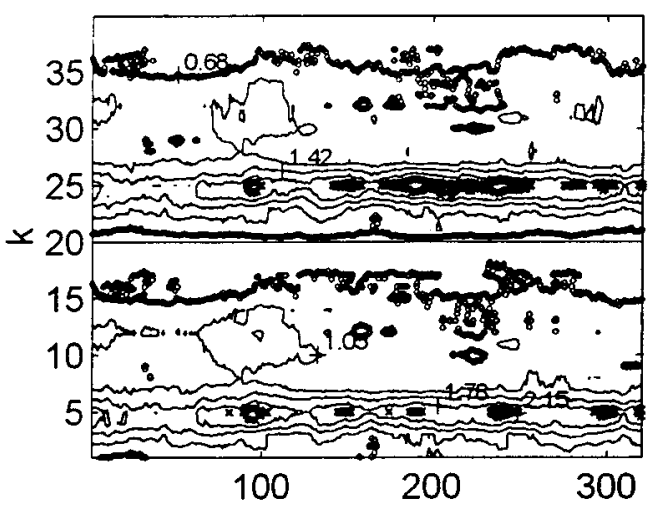

(b) Estimated state vector

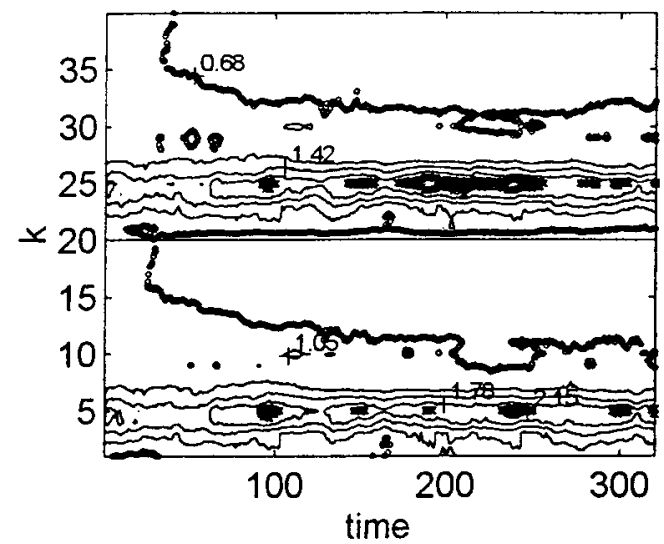

Fig. 8. (Set 2) Contour plots comparing (a) the synthesized optoatmospheric parameters and (b) the EKF estimates.

inversion range becomes limited to $2 \mathrm{~km}$ (eighth observation cell). This hypothesis is corroborated by the contour plot of Fig. 8 and the temporal evolution of the filter's estimates in Fig. 9, where it is seen that the filter loses track of the physical situation from the
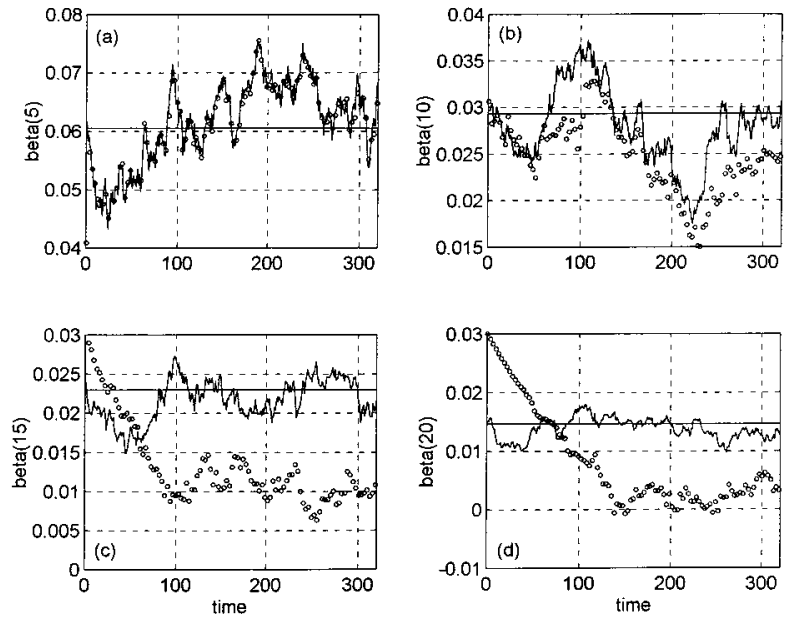

Fig. 9. (Set 2) Temporal evolution of the backscatter profiles in four representative observation cells along the lidar beam path: (horizontal lines) starting backscatter values for the atmospheric simulator, (solid curves) atmospheric backscatter evolution, (circles) EKF estimates. 

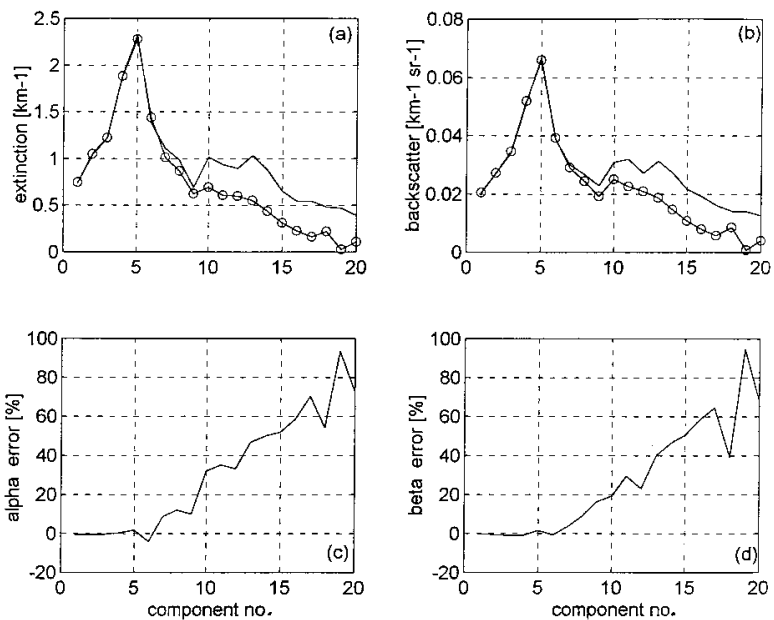

Fig. 10. (Set 2) Inversion results after 320 iterations: (a) final atmospheric extinction (solid curve), final EKF extinction estimates (circles); (b) final atmospheric backscatter (solid curve), final EKF backscatter estimates (circles); (c) extinction relative inversion error; (d) backscatter relative inversion error.

ninth backscatter component up (2292 $\mathrm{m}$ ), where the SNR becomes $21.5 \mathrm{~dB}$. What is more, if in Fig. 8 one follows the isoextinction and isobackscatter contour lines labeled 0.68 (arbitrary units), which correspond to far ranges between 3.3 and $4.5 \mathrm{~km}$, the filter's estimates by chance match the atmospheric parameters at approximately time $t_{k}=40$, pass them by, and eventually become stranded at a wrong estimation. This behavior is clearly illustrated in Fig. 9. Here it is shown that the filter's estimates yield reasonable backscatter inversions until the tenth observation cell, even though the filter tends to become sluggish from this cell up. Performance in the 15th and 20th cells is completely wrong as expected, since at the far ranges the actual measurement received on any particular sample run is hidden by observation noise and does not make any improvement in the filter's estimates. Figure 10 illustrates the final estimates after 320 iterations (along with the expected ones) and the inversion errors, which become higher for this visibility.

As an additional remark, the start-up problem of the filter was especially investigated insofar as it concerns the a priori error covariance matrix $P_{0}^{-}$. In particular, for the first simulation set discussed $\left(V_{M}\right.$ $=39.12 \mathrm{~km}$ ) two extra simulations were carried out, including large modeling errors in the Markovian intensity parameter $p$ [Eq. (28)], which is directly related to the search span of the filter through Eqs. (32) and (33). In the first simulation, we set $p_{a}=$ $50 \%$ and $p=0.5 \%$, and in the second one, $p_{a}=5 \%$ and $p=50 \%$. The results obtained were substantially different: In the first case, where the filter was set with a low $P_{0}^{-}$a sluggish, poor estimation of the extinction component was retrieved, whereas the backscatter profile was estimated reasonably well. In the second, where the filter was set with a $P_{0}^{-}$ higher than the atmospheric state-vector covariance matrix $Q_{k, a}$, both parameters were inverted reasonably well and the filter's estimates do not degrade much from the plots presented so far in Figs. 3-7.

\section{Example of a Real Application}

In this section we tackle the first real application of the filter to the inversion of the sought-after atmospheric optical parameters from power returns measured with an elastic-backscatter lidar system of the Polytechnic University of Catalonia at our premises in Barcelona, Spain, whose main specifications are given in Table 2. Key features of the system are that it is limited to vertical exploration and that an adjustable biaxial arrangement between the laser and the telescope axis is used. In spite of the fact that this complicates system pointing because the telescope can be tilted, when it is combined with a lownoise wide-variable-responsivity receiver, both shortand far-range exploration is possible.

In the measurement campaign of 25 September 1996, the telescope was aimed at far-range exploration

Table 2. Lidar System Specifications

\begin{tabular}{|c|c|c|c|c|c|}
\hline & \multirow{2}{*}{$\frac{\text { Laser }}{\text { Nd:YAG }}$} & \multirow{2}{*}{\multicolumn{2}{|c|}{$\frac{\text { Receiver }}{2 \mathrm{~m}}$}} & \multicolumn{2}{|c|}{ Main System Specifications } \\
\hline $\begin{array}{l}\text { Gain } \\
\text { medium }\end{array}$ & & & & Vertical biaxial & \\
\hline \multirow[t]{2}{*}{ Energy } & $500 \mathrm{~mJ}\left(\lambda_{1}=532 \mathrm{~nm}\right)$ & Aperture & $20-\mathrm{cm}$ diameter & $\begin{array}{l}\text { System noise-equivalent } \\
\text { power }\end{array}$ & $70 \mathrm{fW} \mathrm{Hz}^{-1 / 2}$ \\
\hline & $1 \mathrm{~J}\left(\lambda_{2}=1064 \mathrm{~nm}\right)$ & Field of view & $0.2 \mathrm{mrad}$ & $\begin{array}{l}\text { Minimum detectable } \\
\text { power }\end{array}$ & $<5 \mathrm{nW}$ \\
\hline Divergence & $0.1 \mathrm{mrad}$ & & & & \\
\hline \multirow[t]{5}{*}{ Pulse Length } & $\approx 10 \mathrm{~ns}$ & Detector & Avalanche photodiode & Acquisition & $20 \mathrm{Msps} / 12 \mathrm{bits}^{a}$ \\
\hline & & Detector gain & $M=1$ to 400 & Range resolution & $7.5 \mathrm{~m}$ \\
\hline & & $\begin{array}{l}\text { Detector } \\
\text { responsivity }\end{array}$ & $0.24 \mathrm{~A} / \mathrm{W}(M=1)$ & Time resolution & $50 \mathrm{~ns}$ \\
\hline & & $\begin{array}{l}\text { Net respon- } \\
\text { sivity }\end{array}$ & $6 \times 10^{1}-3 \times 10^{6} \mathrm{~V} / \mathrm{W}$ & $\begin{array}{l}\text { Pulsed repetition } \\
\text { frequency }\end{array}$ & $10 \mathrm{~Hz}$ \\
\hline & & Bandwidth & $10 \mathrm{MHz}$ & & \\
\hline
\end{tabular}

${ }^{\mathrm{a}} \mathrm{Msps}$ is $10^{6}$ samples per second. 

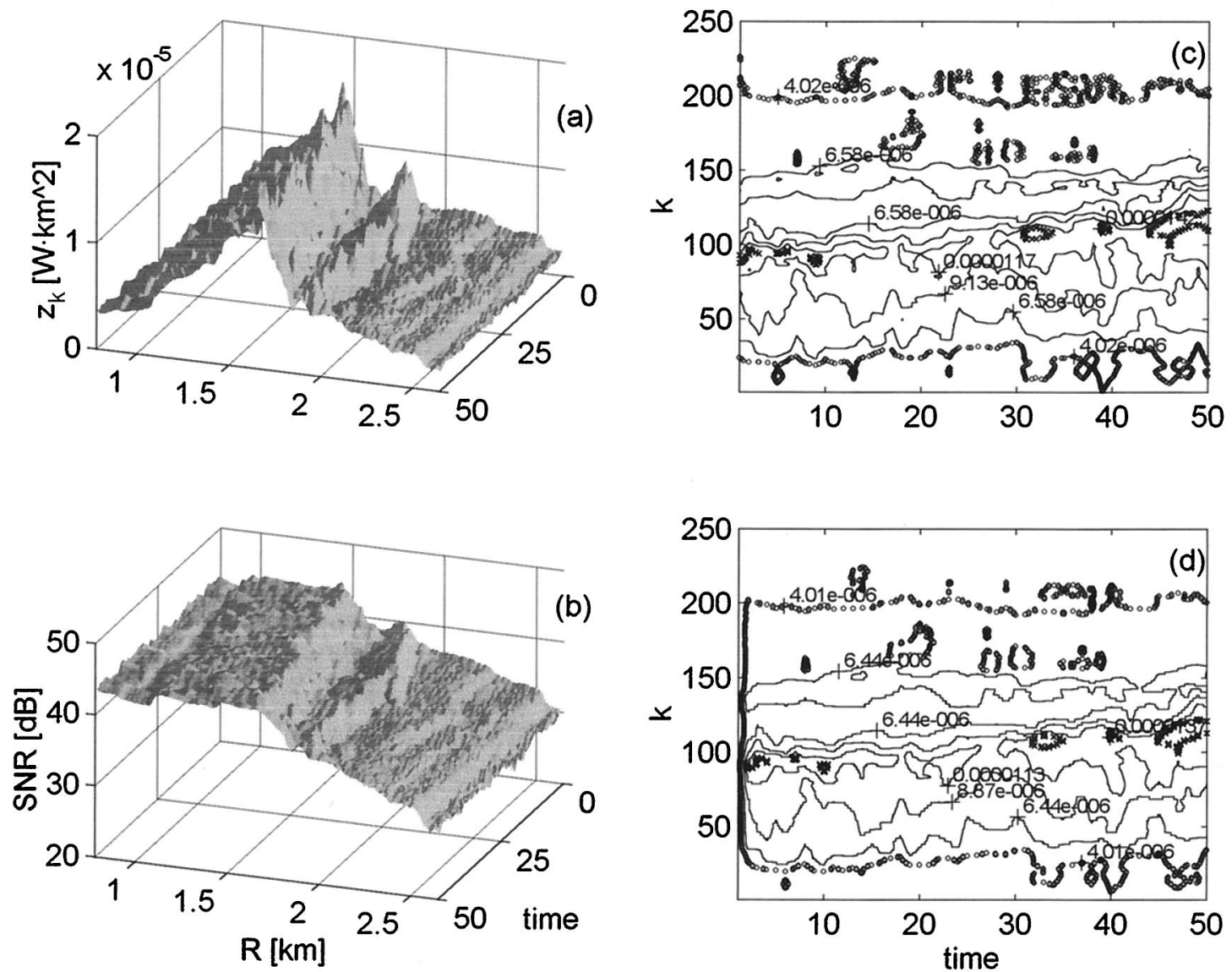

Fig. 11. Example of a real application: (a) range-corrected received power as observables to the filter $\left[z_{k}=R^{2} P(R)\right]$; the plot consists of 50 signal packets (15 pulses per packet) in the range $0.78-2.65 \mathrm{~km}$; (b) associated SNR at the receiver output; (c) contour plot of (a) after distance is translated into samples (each sample equals $7.5 \mathrm{~m}$ ); by columns, we read the measurement vector $z_{k}$ at successive times $\left(t_{k}=\right.$ $1, \ldots, 50)$; (d) same as (c) but showing EKF range-corrected estimated power during the first 50 iterations.

$(>1-15 \mathrm{~km})$. The visibility was very clear $\left(0.01 \mathrm{~km}^{-1}\right.$ $\lesssim \alpha \lesssim 0.2 \mathrm{~km}^{-1}$ on the basis of visual inspection and Refs. 23 and 24) in the inversion interval of interest, and two high cloud layers were detected at $6.4-7.7-\mathrm{km}$ and 8.6-9.4-km height. In the example presented next, we selected the tropospheric interval between 782.5- and 2650-m height for inversion, which comprises 250 power samples (equal to the filter order), so as to test the filter with an atmospheric scene similar to the first set of simulations of Section 4. This serves the purpose of discussing the EKF performance under nonideal data with regard to the estimation statistics modeled rather than studying atmospheric features such as mixing depth or turbidity.

The system was operated at $390-\mathrm{mJ}$ output energy, 532 -nm wavelength, and $1.8 \times 10^{4}-\mathrm{V} / \mathrm{W}$ net responsivity, which yields the system constant and the noise parameters shown in Table 3. Clean data along with the related SNR are shown in Figs. 11(a)-11(c). This includes both range correction and signal averaging of 15 pulses (which makes sense thanks to the relative stationarity of the received returns). In the plots both temporal and spatial correlation is clearly evident. Range-corrected power estimated during

Table 3. Inversion Parameters for Section 5

$\begin{array}{ll}\text { Basic parameters } & \\ \text { Inversion range [Eq. (9)] } & R_{\min }=782.5 \mathrm{~m}, R_{\max }=2.65 \mathrm{~km}, \Delta R=7.5 \mathrm{~m}[\mathrm{Eq} . \text { (8)] } \\ \text { Order parameters } & N=250, M=2[\mathrm{Eqs} .(11)], \text { iterations }=200 \\ \text { System constant [Eq. (1)] } & A=1.81 \times 10^{-3} \mathrm{~W} \mathrm{~km} \mathrm{~km}^{3} \\ \quad & a=3.7 \times 10^{-9} \mathrm{~W}, b=3.4 \times 10^{-16} \mathrm{~W}^{2} \\ \text { Noise parameters [Eq. (22)] } & \\ \text { Model parameters [Eqs. (28)-(32)] } & p=10 \%, L_{c}=50, \rho=0.3, \rho^{\prime}=0.9 \\ \quad \text { EKF } & P_{0}^{-}=100 Q_{0}, \hat{x}_{0}^{-}=(\alpha, \ldots, \alpha, \beta, \ldots, \beta) \text { (see the text) } \\ \text { Initialization } & \end{array}$


the first 50 iterations of the EKF is shown in Fig. $11(\mathrm{~d})$.

To perform the inversion, the original real data, which contain 50 returns per 250 samples each, are fed to the EKF in a round-robin fashion so that the EKF runs 200 iterations (i.e., four rounds of 50 returns each). Covariance matrices $R_{k}, Q_{k}$, and $P_{k}$ were modeled as follows:

The noise covariance matrix $R_{k}$ was estimated from Eqs. (21) and (22) and complete characterization of the equivalent-noise parameters $a$ and $b$ (Table 3) of the optoelectronic receiver stage. To corroborate this result, the equivalent-noise standard deviation $\sigma_{r}(R)$ was checked with its direct computed counterpart from plot analyses of the recorded voltage set at different ranges along the inversion interval.

Modeling the state-vector covariance matrix $Q_{k}$ was done in two steps: first the intensity parameter $p$ and second the correlation parameters $L_{c}, \rho$, and $\rho^{\prime}$. From Eqs. (28)-(31) it follows that the intensity parameter plays a prominent role in the covariance matrix $Q_{k}$ and hence on the filter search span. To guess $p$, percentage drift in an optical parameter (say, the backscatter) must be estimated [Eq. (28)]. This was done by computing the standard deviation of the range-corrected returns of Fig. 11(a) for each observation cell (i.e., 50-sample length realizations were considered for each cell along the inversion spatial range). As the visibility is very clear, Fig. 11(a) yields itself a backscatter estimation. Were if not for that, such plots would have to be corrected by some approximation of the optical depth for each observation cell. This approach gave standard deviations ranging from $5 \%$ to $22 \%$ along the inversion range considered, and hence $10 \%$ was accepted as a reasonable figure for $p$.

As for the second group of parameters, they were set as follows: Because Fig. 11(a) does not show significantly increasing or decreasing slopes in time series, similar to Fig. 6 (but now for the rangecorrected power instead of the backscatter), $L_{c}$ was set to 50. Another approach is to set $L_{c}$ to some predetermined typical value and, since from Eq. (32) the correlation length translates into a scale factor for $Q_{k}$, compare both measured and EKF estimated power and adjust the intensity parameter $p$ by trial and error. Advice on adjustment of correlation coefficients $\rho$ and $\rho^{\prime}$ has already been given in Section 4, and the figures used are given in Table 3. The extinction being the most sensitive parameter, $\rho$ was set to the low typical range to decorrelate the backscatter from extinction errors that are due to misestimation of the intensity parameter $p$.

The EKF was initialized as above with a uniform state vector $\hat{x}_{0}^{-}$as indicated in Table $3\left(\alpha=10^{-2} \mathrm{~km}^{-1}\right.$ and $\beta=10^{-3} \mathrm{~km}^{-1} \mathrm{sr}^{-1}$ ), and later the inversion was repeated with $\hat{x}_{0}^{-}$at 1 order of magnitude lower. The a priori error covariance matrix $P_{0}^{-}$was conservatively set to $100 Q_{0}[\mu=100$ in Eq. (33)], because it was desired that the filter open as much as possible its initial search span. In both cases identical re- sults, shown in Figs. 12 and 13 (to be explained next), were obtained.

To shed more light on the choice of statistical parameters $\rho, \rho^{\prime}$, and $p$, different sets of parametric inversions sweeping the typical ranges introduced in Section 4 were conducted. In other words, once it was shown that the EKF approach achieved fairly acceptable results when the assumed statistical properties of $Q_{k}$ were only a suitable guess of the hidden atmospheric ones, we decided to investigate its performance in response to poorly guessed statistics in $Q_{k}$ and to determine which warning indicators were available. After careful cross examination of the results, two important warning indicators were found: (1) comparison between the range-corrected received power and the filter's estimated one or, equivalently, the relative error between $z_{k}$ and $\hat{z}_{k}^{-}$[Fig. 12(a)] and (2) time evolution of the $P_{k}, Q_{k}$, and $R_{k}$ traces [Fig. 12(b)]. This enables us to identify both modeling errors and convergence times. Thus, if the statistics
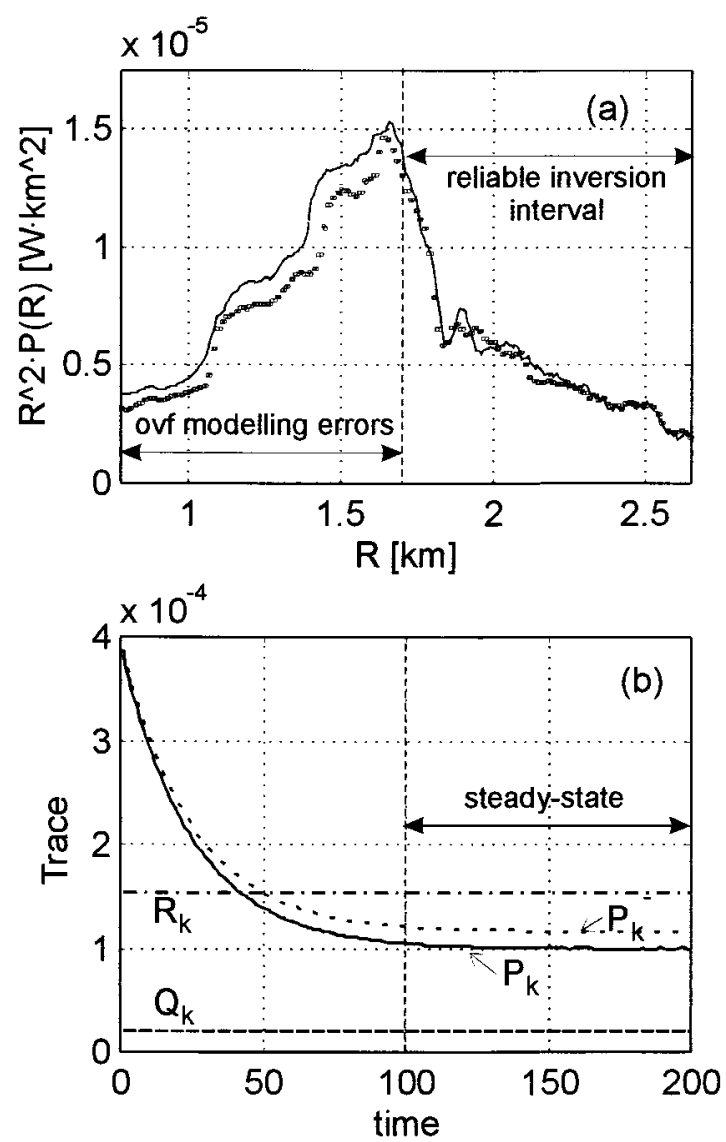

Fig. 12. Identification of modeling errors and convergence times. (a) Comparison between the range-corrected received power (solid curve) and the filter's estimate (circles): The range interval approximately between 0.78 and $1.7 \mathrm{~km}$ is largely affected by ovf modeling errors as discussed in the text, whereas the end interval between 1.7 and $2.65 \mathrm{~km}$ shows much better agreement between both the EKF estimates and the received signal. (b) Time evolution of $P_{k}, P_{k}^{-}, Q_{k}$, and $R_{k}$ traces: Steady convergence is indicated by a constant value in the trace of $P_{k}$ between the traces of $R_{k}$ (measurement noise) and $Q_{k}$ (state-vector noise). 

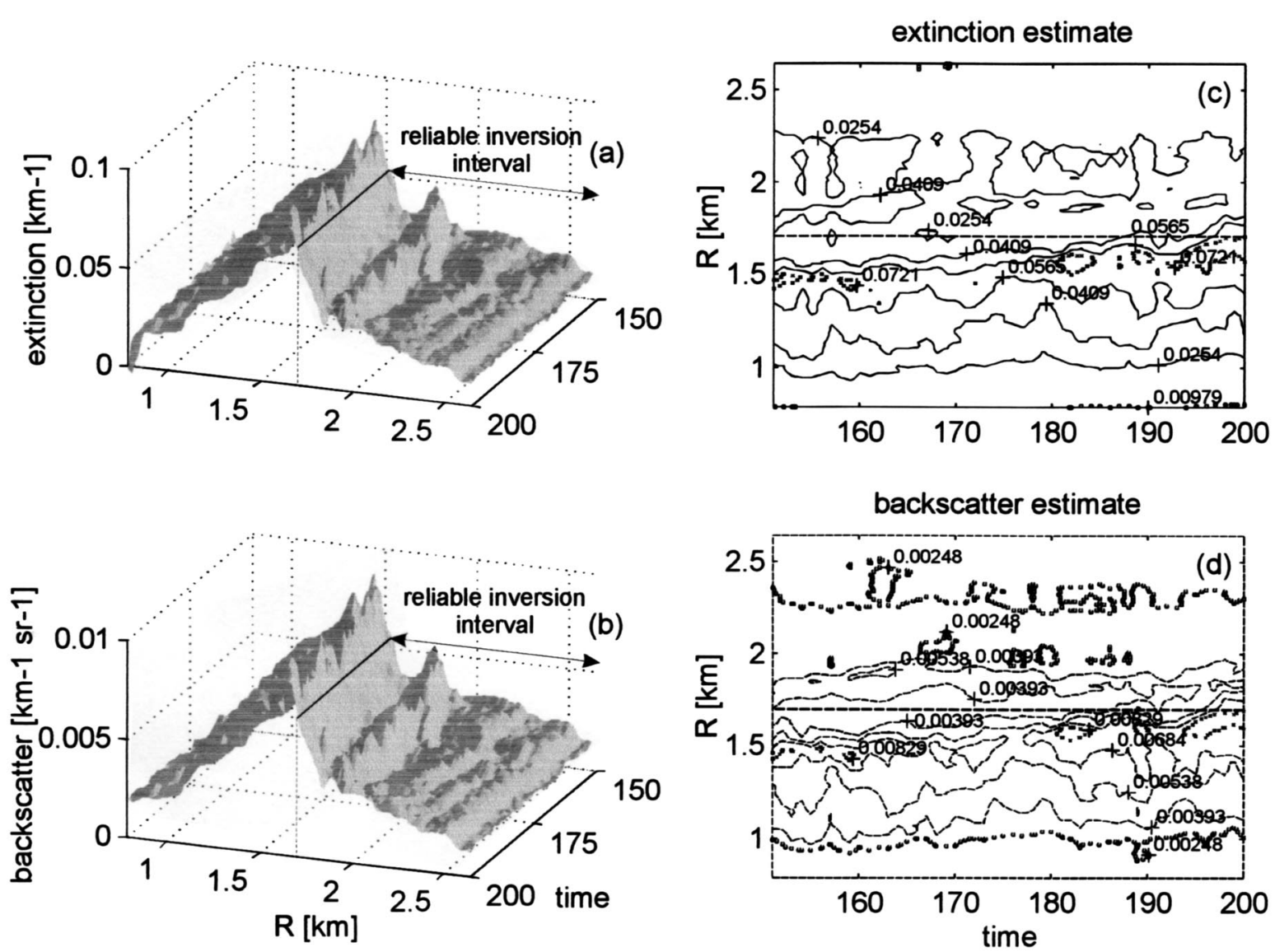

Fig. 13. Inversion results: (a) three-dimensional plot showing the time evolution of the extinction estimates of the filter during the last 50 iterations in response to Figs. 11(a) and 11(b); the reliable inversion interval is virtually free from ovf modeling errors as discussed in the text; (b) same as (a) but showing backscatter estimates; (c) contour plot of (a) in units of $\mathrm{km}^{-1}$; the reliable inversion interval (horizontal dashed line) extents along the vertical of the instrument roughly from $1.7 \mathrm{~km}$ up; (d) same as (c) but for the backscatter estimates in units of $\mathrm{km}^{-1} \mathrm{sr}^{-1}$.

modeled in $Q_{k}$ become poorer, so will power matching. This also provides a tool for fine-tuning the above-mentioned parameters, in particular the intensity parameter $p$. The study also corroborated that our first guess of $p$ following the guidelines indicated above was correct and that the Table 3 set was fairly accurate.

In turn, this enables us to infer other sorts of modeling error that do not necessarily have to do with the statistical modeling but apply to the lidar equation [Eq. (1)] itself. As well as the $1.7-2.65-\mathrm{km}$ range interval can be labeled as a reliable inversion interval, the 0.78-1.7-km interval cannot. Close examination of the latter range interval showed that it was corrupted by overlap factor (ovf) losses. ${ }^{2}$ The ovf depends on the overlap of the area of laser irradiation (illuminated cross section at range $R$ ) with the field of view of the receiver optics and hence is itself a rangedependent function $\xi(R)$ that distorts the basic model of Eq. (1).

As stated above, the $P_{k}$ trace [Fig. 12(b)] is a good indicator of steady tracking of the atmospheric fluctuations and illustrates the way in which the filter works: From the first iterations, the filter progressively reduces the $a$ priori estimation error $e_{k}^{-}$[Eq.
(A18)] until the trace of the a priori error covariance matrix becomes stranded somewhere between the traces of the measurement noise and atmospheric noise covariance matrices $\left(R_{k}\right.$ and $Q_{k}$, respectively). Qualitatively, this suggests that the filter is wise enough to counteract observable noise through convenient averaging with prior estimates but eventually becomes stranded above some state noise that is due to the atmosphere dynamics and is represented by the $Q_{k}$ trace.

To sum up, Fig. 13 shows the inversion results achieved during the last 50 iterations of the filter, along with an indication of the reliable inversion interval based on Fig. 12 indicators. Outside the reliable interval, the inversion becomes progressively corrupted by the geometric ovf as long as one moves toward the first observation cell at $R_{\min }=782.5 \mathrm{~m}$, where the EKF tries to compensate ovf losses with a negative extinction lacking any physical significance. Inside the reliable inversion interval $(1.7-2.65 \mathrm{~km})$, which comprises more than 100 samples, the inversion was further checked by inverting data subintervals with 50 and 100 samples, which inside their respective inversion subranges yielded identical results to those shown in Fig. 13 from the 250-order 
filter. The inversion results obtained have been shown to be in good agreement with typical values for vertical exploration reported in the literature..$^{1,2,11}$

\section{Conclusions}

Historically, the inversion of optical parameters, namely, the atmospheric extinction and backscatter, from the return power of an elastic-backscatter lidar has been tackled by using nonmemory algorithms, basically least-squares curve-fitting procedures and the well-known Klett's method. Through extensive simulation under simplified modeling conditions and a first application to the inversion of real data, it has been shown that an extended Kalman filter (EKF) formulation enables one to invert both extinction and backscatter optical parameters as time, rangedependent functions from the lidar return power. This is a first step in the pioneering field of adaptive joint estimation of the sought-after optical parameters by using an EKF.

The approach presented here replaces tight correlation relations used thus far, such as analytical relationships between extinction and backscatter parameters plus boundary calibrations and the homogeneity hypothesis for the atmosphere, by appropriate correlation models (both temporal and spatial) and the description of the optical parameters as vector stochastic processes. These models try to describe the macroscopic short-term fluctuations of the atmospheric optical parameters (i.e., to model their magnitude variations) through a set of statistical parameters, given as model inputs. The core of the temporal correlation model is that actual temporal variations in the magnitude of the extinction and backscatter parameters have finite correlation length. As to the spatial correlation model, a sensible straightforward correlation graph is used as a rough description of correlation links among the observation cells along the lidar exploration path. Since the estimation criterion is minimum mean square error (in the statistical sense) for the EKF and not the square error as is customary in nonmemory algorithms, the EKF accounts for any prior knowledge through a recursive process in the most convenient way.

The feasibility of the proposed adaptive algorithm has been tested in two ways: First, simulations have been conducted to show that even under moderate modeling errors between the simulated atmospheric model and the EKF one, inversion errors strongly depend on the atmospheric condition (i.e., the visibility) and the signal-to-noise ratio (SNR) along the exploration path (SNR's above $25 \mathrm{~dB}$ along the inversion path have yielded relative inversion errors in the optical parameters below $20 \%$ for visual ranges of approximately $4 \mathrm{~km}$ ). The atmospheric extinction becomes the most difficult parameter to invert because of its sensitivity to power misestimation. Second, we have broken through the idealness that any set of simulated data conveys with regard to the atmospheric model of the filter, and the EKF has tackled the inversion of a similar atmospheric scene, but this time from real data, with successful results.
In addition, both the power mismatch and the $a$ priori error covariance matrix trace $\left(P_{k}\right)$ have been shown to be good performance indicators on account of modeling errors.

All things considered, the EKF promises to be a useful tool in the field of joint estimation of the atmospheric extinction and backscatter optical parameters from elastic-backscatter lidar returns.

\section{Appendix A: Review of the Extended Kalman Filter and Notation Used}

Some of the most successful applications of the Kalman filter are obvious for situations for which nonlinear dynamics and/or measurements must be considered.

The system model to estimate is a stochastic discrete time vector $x_{k}$ modeled by

$$
x_{k+1}=f\left(x_{k}\right)+w_{k},
$$

where $f_{k}$ is a nonlinear function that relates the vector state from time $t_{k}$ to time $t_{k+1}$ and $w_{k}$ is zero-mean, white Gaussian noise having a covariance matrix

$$
Q_{k}=E\left(w_{k} w_{k}^{\mathrm{T}}\right) \text {. }
$$

The measurement model is formed by the observable vector $z_{k}$, so that observation takes place at discrete times $t_{k}$, according to the following relationship:

$$
z_{k}=h_{k}\left(x_{k}\right)+v_{k},
$$

where $h_{k}$ is a nonlinear function of the state $x_{k}$ and $v_{k}$ is zero-mean, white Gaussian noise having a covariance matrix

$$
R_{k}=E\left(v_{k} v_{k}^{\mathrm{T}}\right) .
$$

In addition, the noisy vectors $v_{k}$ and $w_{k}$ must be uncorrelated. In instances where these conditions are not fulfilled, it is possible to define an augmented state vector and estimate the correlated noisy samples $w_{k}^{\prime}$ and $v_{k}^{\prime}$ from others, $w_{k}$ and $v_{k}$, that form an uncorrelated, orthogonal base.

At this point it is assumed that an initial estimate of the process at the same point in time, $t_{k}$, is known. This estimation, is called the $a$ priori estimate and is denoted $\hat{x}_{k}^{-}$, where the superscript minus sign is a reminder that it is the best estimate before assimilation of the measurement at $t_{k}$. The a posteriori (updated) estimate is denoted as $\hat{x}_{k}$.

Using these definitions and simple Taylor-series expansion of Eqs. (A1) and (A3) around the a posteriori $\hat{x}_{k}$ and $a$ priori $\hat{x}_{k}^{-}$estimates and retaining only first-order terms, as is customary, we find that

$$
\begin{aligned}
& f_{k}\left(x_{k}\right) \approx f_{k}\left(\hat{x}_{k}\right)+\left.\frac{\partial f_{k}(x)}{\partial x}\right|_{x=\hat{x}_{k}}\left(x_{k}-\hat{x}_{k}\right), \\
& h_{k}\left(x_{k}\right) \approx h_{k}\left(\hat{x}_{k}^{-}\right)+\left.\frac{\partial h_{k}(x)}{\partial x}\right|_{x=\hat{x}_{\bar{k}}^{-}}\left(x_{k}-\hat{x}_{k}^{-}\right) .
\end{aligned}
$$


These expressions bridge the gulf with the classical linear filter if the equivalent matrices $F_{k}$ and $H_{k}$ are defined in the following way:

$$
\begin{aligned}
& F_{k}=\left.\frac{\partial f_{k}(x)}{\partial x}\right|_{x=\hat{x}_{k}}, \\
& H_{k}=\left.\frac{\partial h_{k}(x)}{\partial x}\right|_{x=\hat{x_{k}}} .
\end{aligned}
$$

Identification with the first-order terms of approximations (A5) and (A6) yields

$$
\begin{aligned}
x_{k+1} & \approx f_{k}\left(\hat{x}_{k}\right)+F_{k}\left(x_{k}-\hat{x}_{k}\right)+w_{k}, \\
z_{k} & \approx h_{k}\left(\hat{x}_{k}^{-}\right)+H_{k}\left(x_{k}-\hat{x}_{k}^{-}\right)+v_{k} .
\end{aligned}
$$

Approximations (A9) and (A10) represent the linearized version of the filter and resemble those of a linear Kalman filter except for the fact that rather than presenting total quantities to the filter, we consider incremental ones. In relation to approximations (A9) and (A10), these are

$$
\begin{aligned}
& \Delta x_{k}=x_{k+1}-f_{k}\left(\hat{x}_{k}\right), \\
& \Delta z_{k}=z_{k}-h_{k}\left(\hat{x}_{k}^{-}\right) .
\end{aligned}
$$

In summary the EKF's recursive equation set becomes

$$
\begin{aligned}
\hat{x}_{k} & =\hat{x}_{k}^{-}+K_{k}\left[z_{k}-h_{k}\left(\hat{x}_{k}^{-}\right)\right], \\
P_{k} & =\left(I-K_{k} H_{k}\right) P_{k}^{-}, \\
\hat{x}_{k+1}^{-} & =f_{k}\left(\hat{x}_{k}\right), \\
P_{k+1}^{-} & =F_{k} P_{k} F_{k}^{\mathrm{T}}+Q_{k}, \\
K_{k} & =P_{k}^{-} H_{k}^{\mathrm{T}}\left(H_{k} P_{k}^{-} H_{k}^{\mathrm{T}}+R_{k}\right)^{-1},
\end{aligned}
$$

where $K_{k}$ is the Kalman gain and $P_{k}^{-}$is the associated error covariance matrix, defined as

$$
P_{k}^{-}=E\left(e_{k}^{-} e_{k}^{-\mathrm{T}}\right)=E\left[\left(x_{k}-\hat{x}_{k}^{-}\right)\left(x_{k}-\hat{x}_{k}^{-}\right)^{\mathrm{T}}\right],
$$

where $e_{k}^{-}$is the $a$ priori estimation error.

Yet, careful attention should be drawn to the fact that use of the EKF is risky, as the linearization process takes places about the filter's estimated trajectory of the state vector rather than about a precomputed nominal trajectory. That is, the partial derivatives are evaluated along a trajectory that has been updated with the filter's estimates; thus it depends on the measurements. As a result, the filter is more likely to diverge.

In the $\mathrm{EKF}$ problem formulated in this work, the system model is linear and the state-space representation of the atmospheric state vector is given by

$$
x_{k+1}=\Phi_{k} x_{k}+w_{k}
$$

where $\Phi_{k}$ is the transition state matrix from time $t_{k}$ to time $t_{k+1}$. If both the system and the observation model are linear, Eqs. (A13)-(A17) become the same after we replace $F_{k}$ by $\Phi_{k}$ and $h_{k}$ by $H_{k}$.
We acknowledge the sponsorship of the CICYT (Interministry Committee for Science and Technology) under grant AMB96-1144-C02-C01.

\section{References}

1. R. T. H. Collis and P. B. Russell, "Laser measurement of particles and gases by elastic backscattering and differential absorption," in Laser Monitoring of the Atmosphere, E. D. Hinkley, ed. (Springer-Verlag, New York, 1976), Chap. 4, pp. 91-102.

2. R. M. Measures, "Laser-remote-sensor equations," in Laser Remote Sensing: Fundamentals and Applications (Krieger, Malabar, Fla., 1992), Chap. 7, pp. 237-280.

3. D. K. Killinger and N. Menyuk, "Laser sensing of the atmosphere," Science 235, 37-45 (1987).

4. A. I. Carswell, "Lidar remote sensing of atmospheric aerosols," in Propagation Engineering: Third in a Series, L. R. Bissonnette and W. B. Miller, eds., Proc. SPIE 1312, 206-220 (1990).

5. G. J. Kunz and G. de Leeuw, "Inversion of lidar signals with the slope method," Appl. Opt. 32, 3249-3256 (1993).

6. J. D. Klett, "Stable analytical inversion solution for processing lidar returns," Appl. Opt. 20, 211-220 (1981).

7. R. J. Barlow, Statistics (Wiley, New York, 1989).

8. J. J. More, "The Levenberg-Marquardt algorithm: implementation and theory," in Numerical Analysis, Lecture Notes in Mathematics 630, G. A. Watson, ed. (Springer-Verlag, New York, 1977), pp. 105-116.

9. J. D. Klett, "Lidar calibration and extinction coefficients," Appl. Opt. 22, 514-515 (1983).

10. J. D. Klett, "Lidar inversion with variable backscatter/extinction ratios," Appl. Opt. 24, 1638-1643 (1985).

11. G. J. Kunz, "Probing of the atmosphere with lidar," in Proceedings of Remote Sensing of the Propagation Environment (AGARD-CP-502), 23, 1-11 (1992).

12. R. E. Kalman, "A new approach to linear filtering and prediction problems," J. Basic Eng. 82, 35-46 (1960).

13. H. W. Sorenson, Kalman Filtering Techniques. Advances in Control Systems. Theory and Applications (IEEE, New York, 1985), Vol. 3.

14. R. G. Brown and P. Y. C. Hwang, Introduction to Random Signals and Applied Kalman Filtering (Wiley, New York, 1992).

15. B. J. Rye and R. M. Hardesty, "Nonlinear Kalman filtering techniques for incoherent backscatter lidar: return power and log power estimation,” Appl. Opt. 28, 3908-3917 (1989).

16. D. G. Lainiotis, P. Papaparaskeva, G. Kothapalli, and K. Plataniotis, "Adaptive filter applications to LIDAR: return power and log power estimation," IEEE Trans. Geosci. Remote Sens. 34, 886-891 (1996).

17. R. J. McIntyre, "Multiplication noise in uniform avalanche photodiodes," IEEE Trans. Electron Devices ED-13, 164-168 (1966).

18. P. S. Maybeck, Stochastic Models, Estimation and Control (Academic, New York, 1977), Vol. 1.

19. W. B. Jones, Introduction to Optical Fiber Communication Systems (Holt, Rinehart \& Winston, New York, 1988), Chap. 7, 8.

20. A. Papoulis, Probability, Random Variables and Stochastic Processes (McGraw-Hill, New York, 1991).

21. D. G. Lainiotis, "Partitioned estimation algorithms. I: Nonlinear estimation,” J. Inf. Sci. 7, 203-255 (1974).

22. D. G. Lainiotis, "Partitioning: a unifying framework for adaptive systems. I: Estimation," Proc. IEEE 64, 11261143 (1976).

23. H. Koshmieder, "Theorie der Horizontalen Sichtweite," Beitr. Phys. Freien Atmos. 12, 33-53 (1924).

24. P. W. Kruse, L. D. McGlauchlin, and R. B. McQuiston, Elements of Infrared Technology: Generation, Transmission and Detection (Wiley, New York, 1962). 